\title{
A Tale of Two Hypersecreting Adrenal Neoplasms in the Heartland of COVID-19 Pandemic, Lombardy, Italy
}

\author{
Benedetta Zampetti $\mathbb{D}^{\mathrm{D}},{ }^{1}$ Roberto Attanasio ${ }^{\mathrm{D}} \mathrm{,},{ }^{2}$ Emanuela Carioni, ${ }^{1}$ Daniela Dallabonzana, ${ }^{1}$ \\ Iuliana Pauna, ${ }^{3}$ Marco Boniardi, ${ }^{3}$ and Renato Cozzi $\mathbb{D}^{2}$ \\ ${ }^{1}$ ASST Grande Ospedale Metropolitano Niguarda, Endocrinology Department, Milan, Italy \\ ${ }^{2}$ Scientific Committee Associazione Medici Endocrinologi, International Chapter of Clinical Endocrinology, Milan, Italy \\ ${ }^{3}$ ASST Grande Ospedale Metropolitano Niguarda, General Oncologic and Mini-invasive Surgery Department, Milan, Italy
}

Correspondence should be addressed to Benedetta Zampetti; benedettazampetti@yahoo.it

Received 8 November 2021; Accepted 26 January 2022; Published 18 February 2022

Academic Editor: J. Paul Frindik

Copyright (c) 2022 Benedetta Zampetti et al. This is an open access article distributed under the Creative Commons Attribution License, which permits unrestricted use, distribution, and reproduction in any medium, provided the original work is properly cited.

In this study, we report the management, in Lombardy, Italy, of one patient with Cushing's syndrome due to adrenal adenoma and another one with pheochromocytoma, whose surgeries were deferred owing to the COVID-19 pandemic.

\section{Introduction}

Surgery is the mainstay of treatment for hypersecreting adrenal lesions, whenever clinically and technically feasible $[1,2]$.

COVID-19 pandemic obliged physicians to find out alternative options to classical pathways, in order to lower viral spread and related dangers as well as to cope with redeployment of personnel and resources.

We describe here two cases in whom surgery for adrenal Cushing's syndrome (CS) and pheochromocytoma (PHEO) could not be performed as usual and was deferred due to the unavailability of surgical facilities.

\section{Case 1}

A 69-year-old woman was evaluated for CS.

Her history included arterial hypertension for 10 years, hyperlipidemia, and euthyroid multinodular goiter.

In 2017, type 2 diabetes mellitus was diagnosed, and a $35 \mathrm{~mm}$ right adrenal mass was incidentally found at US at another hospital. No endocrine work-up was performed. Due to high density (40 HU) of the lesion, a ${ }^{18}$ F-FDG-PET scan was performed with negative results. The size of the lesion was unchanged at CT follow-up in the following two years.
In December 2019, clinical picture worsened with progressive loss of independence and self-care ability up to walking impairment. Physical examination was suggestive of hypercortisolism (central obesity, muscle atrophy, moon facies, buffalo hump, and leg ulcers). Endocrine work-up showed ACTH-independent hypercortisolism: UFC was $214 \mathrm{nM} / 24 \mathrm{~h}$ (by LC-MS/MS, nv < 168), serum cortisol after overnight $1 \mathrm{mg}$ dexamethasone test was $240 \mu \mathrm{g} / \mathrm{L}(\mathrm{nv}<18)$, and ACTH $1 \mathrm{ng} / \mathrm{L}$ (normal value: $7-63 \mathrm{ng} / \mathrm{L}$ ). Urinary metanephrines were within the normal range. CT scan confirmed a $35 \mathrm{~mm}$ right adrenal mass.

In July 2020, during the full-blown pandemic period, glucometabolic derangement required hospitalization in our hospital. Nasal swab was negative for SARS-CoV-2, fasting glucose was $300 \mathrm{mg} / \mathrm{dL}$, HbA1c $12.5 \%$, eGFR $34 \mathrm{~mL} / \mathrm{min} /$ $1.73 \mathrm{~m}^{2}$, AST $39 \mathrm{U} / \mathrm{L}$, ALT $60 \mathrm{U} / \mathrm{L}$, and gamma-GT $608 \mathrm{U} / \mathrm{L}$. Abdominal CT did not show additional lesions beyond the known $36 \times 33 \mathrm{~mm}$ right adrenal nodule; in particular, the liver and biliary tree were normal. Screening for complications of CS ruled out vertebral fractures and disclosed hypertrophic cardiomyopathy with outflow obstruction.

The whole picture was so severe that she was not considered suitable for surgery that was anyway inaccessible due to pandemic. The impairment in the liver function test (LFT) was attributed to steroid hepatitis. 
Initial daily treatment included bisoprolol $5 \mathrm{mg}$, perindopril $10 \mathrm{mg}$, amlodipine $10 \mathrm{mg}$, doxazosin $4 \mathrm{mg}$, furosemide $50 \mathrm{mg}$, spironolactone $37 \mathrm{mg}$, atorvastatin $20 \mathrm{mg}$, and acetylsalicylic acid $100 \mathrm{mg}$. Antidiabetic treatment was uptitrated with insulin (total daily dose: $84 \mathrm{U}$; degludec, $38 \mathrm{U}$, fast-acting aspart, $14+18+14 \mathrm{U}$ ), dapagliflozin $10 \mathrm{mg} /$ day, and long-acting exenatide $2 \mathrm{mg} /$ week.

Medical treatment with ketoconazole (KCZ) was then cautiously administered, starting with $200 \mathrm{mg}$ /day and then uptitrated to $400 \mathrm{mg} /$ day. The choice of KCZ was due to transient unavailability of metyrapone (osilodrostat was not yet marketed). The patient complained nausea, and KCZ dose was downtitrated to 200 and $400 \mathrm{mg}$ every other day. UFC values progressively decreased to $38 \mu \mathrm{g} / 24 \mathrm{~h}$ (nv 35-137) as well as transaminase and gamma-GT values, and cortisone acetate $12.5 \mathrm{mg} /$ day was added. This block-and-replace schedule was well tolerated, clinical picture improved, and insulin dose was progressively reduced to $70 \mathrm{U} /$ day due to the continuous occurrence of hypoglycemic episodes.

She was discharged home clinically improved, with the indication to weekly control of LFT, insulin dose tapering according to glucose automonitoring and medical advice, and quick glucocorticoid dose increase in case of fever or stress. Adrenal resection should have been planned as soon as possible in relation to clinical improvement and pandemic evolution.

After one month, she was hospitalized in another ward owing to syncope for acute adrenal failure due to symptomatic febrile urinary infection without a timely quick increase of glucocorticoid dosage. The daily block-and-replace schedule was adjusted to KCZ $400 \mathrm{mg}$ plus cortisone acetate $25 \mathrm{mg}$. This treatment induced a progressive amelioration of clinical picture, including healing of leg ulcers, mood improvement, and restoring of the ability to walk. LFT normalized, antihypertensive drug dosage was reduced, HbA1c dropped to $6.2 \%$, and insulin dose was reduced to $26 \mathrm{U} /$ day owing to frequent hypoglycemic episodes. UFC was now normal (ranging 31-52 $\mu \mathrm{g} / 24 \mathrm{~h}$ ).

Due to the persistence of the COVID-19 pandemic in our region and the unavailability of any surgical facilities for not urgent surgeries, despite our pressing on surgeon and hospital management, the patient is still scheduled for adrenal resection. She is carrying on $\mathrm{KCZ}$ plus cortisone acetate therapy at fixed dose, with the advice to quickly increasing the steroid dose as soon as any suspect of impending infection occurs. A progressive improvement in clinical and biochemical picture has been observed on this medical treatment that is aimed to keep a good control of arterial pressure and a fair control of the glucose metabolism while avoiding any hypoglycemia.

In July 2021, abdomen CT scan did not show any change in tumor size; the values of UFC and ACTH were $35 \mu \mathrm{g} / 24 \mathrm{~h}$ and $1.5 \mathrm{ng} / \mathrm{L}$, respectively, on a block-and-replace treatment (KCZ $400 \mathrm{mg}$ plus cortisone acetate $25 \mathrm{mg} /$ day).

\section{Case 2}

An 83-year-old woman was evaluated for cardiogenic shock.

Her history was remarkable for hyperlipidemia, gastroesophageal reflux, right breast cancer (in 2000 with negative annual follow-up), and surgically treated autonomous thyroid adenoma (in 1980). In addition, she had undergone cholecystectomy in 2018 that was complicated by severe hypertensive crisis with acute heart failure.

In September 2019, abdominal plastic surgery was complicated by severe hypertensive crisis with acute pulmonary edema, cardiogenic shock, and acute renal failure. She was thus transferred to ICU for suspected acute coronary syndrome. Multiple noncritical stenoses were disclosed at coronary angiography and reverse Takotsubo at ventriculography. Chest CT disclosed a left adrenal lesion consistent with PHEO. Conservative therapies (ramipril, lercanidipine, atenolol, and furosemide) induced recovery of systolic function (ejection fraction increased from $5 \%$ to $62 \%$ ).

Endocrine work-up confirmed PHEO: urinary adrenaline was $34 \mu \mathrm{g} / 24 \mathrm{~h}(\mathrm{nv}<22)$, noradrenaline $81 \mu \mathrm{g} / 24 \mathrm{~h}$ (nv <85), metanephrines $1932 \mu \mathrm{g} / 24 \mathrm{~h}(\mathrm{nv}<297)$, and normetanephrines $2009 \mu \mathrm{g} / 24 \mathrm{~h} \quad(\mathrm{nv}<354)$. At retrospective inquiring, the patient reported palpitations, tremors, diaphoresis, headaches, anxiety, and panic attacks. Fasting plasma glucose was $79 \mathrm{mg} / \mathrm{dL}$ and HbAlc 5.2\%.

Abdominal CT and MRI confirmed a $45 \times 31 \mathrm{~mm}$ left adrenal lesion, with irregular margins and inhomogeneous contrast enhancement, whose density was $30 \mathrm{HU}$, without other remarkable lesions.

The planning of adrenal resection was postponed due to an adrenal hematoma developed while on LMWE treatment. Pressure control was maintained with doxazosin (in addition to ongoing treatment) titrated up to $8 \mathrm{mg}$ bid. In January 2020, CT showed hematoma reabsorption, but while waiting for scheduled surgery, there was the outbreak of pandemic. The patient was monitored throughout Spring and Summer with frequent telemedicine controls that confirmed optimal pressure control, without any side effect of antihypertensive treatment.

In October 2020, nasal swab was negative for SARS$\mathrm{CoV}-2$, and she underwent laparoscopic left adrenalectomy, taking advantage of a transient decrease of pandemic burden.

Postoperative recovery was uneventful, no hypotension occurred, and the patient was discharged home on atenolol and doxazosin that were quickly tapered and finally discontinued within one month. Histologic examination confirmed the diagnosis of PHEO: the lesion infiltrated the perivisceral adipose tissue due to the previous hematoma, but there was no angioinvasion, Ki-67 was $<1 \%$, and PASS score was 7 .

One month later, urinary metanephrines were normalized, namely, 93 and $282 \mu \mathrm{g} / 24 \mathrm{~h}$ for metanephrine and normetanephrine, respectively, and remained normal at the following control.

A recent $\mathrm{CT}$ did not disclose any tumor remnant or relapse.

\section{Discussion}

CS is a life-threatening disease, with vascular disease and infections representing the most common cause of death, mainly in elderly patients. Both diagnosis and management 
of CS might be cumbersome even in ordinary times, mostly in patients with a cyclic disease. The diagnosis in patient 1 was straightforward but the management was not, due to the COVID-19 pandemic. Surgery is the first-line treatment for CS, regardless of its etiology [1], but in some extremely frail patients, it should be postponed even in ordinary times, in order to ameliorate clinical picture with steroidogenesis inhibitors according to clinical status. Furthermore, surgery should be considered with caution during the pandemic because cortisol hypersecretion may lead to a lessening of immune reaction. In addition, following an issue of hospital management, only urgent cases should be admitted to surgery unit due to the shortage of intensive care units and healthcare personnel.

We followed thus successfully the recommendations of the European Society of Endocrinology [3] that points to surgery only in patients who cannot be controlled with medical therapy or in those requiring biopsy or resection because of possible cancer.

The infective risk is indeed increased by defective innate and adaptive immune response due to chronic glucocorticoid exposure [4]. The increased susceptibility to infection is further increased by visceral obesity, hypertension, and diabetes that are characteristics of the disease and hugely heighten the risk of death. Furthermore, CS patients have high risk of thromboembolic events due to many alterations of coagulation and fibrinolysis that in turn worsen the prognosis in the case of COVID-19 infection. We opted thus for drug treatment to get control of the disease while waiting for more propitious times for a surgical approach.

Among the different steroidogenesis inhibitors, we had to choose KCZ because osilodrostat was not marketed yet, and there was a temporary shortage of metyrapone supply. Keeping in mind the liver toxicity of KCZ, reportedly occurring in 3-19\% of treated patients [5], this choice might have been regarded as hazardous in a patient with severe derangement of LFT. Anyway, the severity of clinical picture prompted us to start treatment with a tight monitoring. Treatment obtained actually a quick and progressive improvement in clinical and biochemical picture. No escape was observed, on the contrary adrenal failure developed prompting the use of a block-and-replace schedule. This option has proved effective beyond all our expectations.

In patient 2, the diagnosis of PHEO was neglected for a long time, until catastrophic acute worsening of cardiac function occurred that required intensive treatment.

According to Endocrine Society guidelines, the treatment for PHEO is surgical adrenalectomy after a brief period of medical preparation (7-14 days) with alpha-adrenergic receptor blockade to minimize perioperative complications [2].

The unusual circumstances created by the pandemic necessitated a more prolonged preoperative period of medical management, as already described in other 4 cases recently reported [6,7]. At last, the adrenal tumor was successfully resected after a prolonged pharmacologic control of blood pressure, and the patient is doing well off antihypertensive treatment.
This effort of progressive continuous adjustment should always take into account both the epidemiological environment with its constraints and the individual clinical picture.

\author{
Abbreviations \\ ACTH: Adrenocorticotropic hormone \\ ALT: Alanine transaminase \\ AST: $\quad$ Aspartate transaminase \\ COVID-19: Corona-virus disease 2019 \\ CS: Cushing's syndrome \\ CT: Computerized tomography \\ eGFR: Estimated glomerular filtration rate \\ gamma- Gamma-glutamyl-transferase \\ GT: \\ HbA1c: Glycated hemoglobin \\ HU: Hounsfield units \\ KCZ: Ketoconazole \\ LC-MS/ Liquid chromatography-tandem mass \\ MS: $\quad$ spectrometry \\ LFT: $\quad$ Liver function test \\ LMWE: Low molecular weight heparin \\ MRI: $\quad$ Magnetic resonance imaging \\ Nv: Normal values \\ PASS: $\quad$ Pheochromocytoma of the Adrenal Gland \\ PET: $\quad$ Positron emission tomography \\ PHEO: Pheochromocytoma \\ UFC: $\quad$ Urinary free cortisol \\ US: Ultrasonography.
}

\section{Consent}

The patients have given informed consent for the publication of their medical history.

\section{Conflicts of Interest}

The authors declare that they have no conflicts of interest.

\section{References}

[1] L. K. Nieman, B. M. K. Biller, J. W. Findling et al., "Treatment of Cushing's syndrome: an endocrine society clinical practice guideline practice guideline," Journal of Clinical Endocrinology \& Metabolism, vol. 100, no. 8, pp. 2807-2831, 2015.

[2] J. W. M. Lenders, Q.-Y. Duh, G. Eisenhofer et al., "Endocrine Society. Pheochromocytoma and paraganglioma: an Endocrine Society clinical practice guideline," Journal of Clinical Endocrinology \& Metabolism, vol. 99, no. 6, pp. 1915-1942, 2014.

[3] J. Newell-Price, L. K. Nieman, M. Reincke, and A. Tabarin, "Endocrinology in the time of COVID-19: management of Cushing's syndrome," European Journal of Endocrinology, vol. 183, no. 1, pp. G1-G7, 2020.

[4] V. Guarnotta, R. Ferrigno, M. Martino et al., "Glucocorticoid excess and COVID-19 disease," Reviews in Endocrine \& Metabolic Disorders, vol. 22, no. 4, pp. 703-714, 2020.

[5] R. Pivonello, M. De Leo, A. Cozzolino, and A. Colao, "The treatment of Cushing's disease," Endocrine Reviews, vol. 36, no. 4, pp. 385-486, 2015. 
[6] E. A. Japp, A. Leiter, E. A. Tsomos, S. A. Reda, and A. C. Levine, "Management of 3 cases of pheochromocytoma during the COVID-19 pandemic in New York City: lessons learned," Journal of the Endocrine Society, vol. 5, no. 2, pp. 1-6, 2021.

[7] R. Yu, "Successful management of pheochromocytoma and thyroiditis with fever by telemedicine during the COVID-19 pandemic," Endocrine Practice, vol. 26, no. 9, pp. 1042-1043, 2020. 